\title{
Molecular mechanism of point mutation-induced Monopolar spindle 1 (Mps1/TTK) inhibitor resistance revealed by a comprehensive molecular modeling study
}

\author{
Yan Han Corresp.. ${ }^{1}$, Yungang Wu ${ }^{1}$, Yi Xu ${ }^{2}$, Wentao Guo ${ }^{3}$, Na Zhang ${ }^{1}$, Xiaoyi Wang ${ }^{1}$ \\ ${ }^{1}$ Department of TCM Orthopedics \& Traumatology, the First Affiliated Hospital of Wenzhou Medical University, Wenzhou, Zhejiang, China \\ 3 School of Pharmacy, Wenzhou Medical University, Wenzhou, Zhejiang, China \\ Corresponding Author: Yan Han \\ Email address: hanyan@wzhospital.cn
}

Background. Monopolar spindle 1 (Mps1/TTK) is an apical dual-specificity protein kinase in the spindle assembly checkpoint (SAC) that guarantees accurate segregation of chromosomes during mitosis. High levels of Mps1 are found in various types of human malignancies, such as glioblastoma, osteosarcoma, hepatocellular carcinoma, and breast cancer. Several potent inhibitors of Mps1 exist, and exhibit promising activity in many cell cultures and xenograft models. However, resistance due to point mutations in the kinase domain of Mps1 limits the therapeutic effects of these inhibitors. Understanding the detailed resistance mechanism induced by Mps1 point mutations is therefore vital for the development of novel inhibitors against malignancies.

Methods. In this study, conventional molecular dynamics (MD) simulation and Gaussian accelerated MD (GaMD) simulation were performed to elucidate the resistance mechanisms of Cpd-5, a potent Mps1 inhibitor, induced by the four representative mutations I531M, I598F, C604Y, S611R.

Results. Our results from conventional MD simulation combined with structural analysis and free energy calculation indicated that the four mutations weaken the binding affinity of Cpd-5 and the major variations in structural were the conformational changes of P-loop, A-loop and $\alpha \mathrm{C}$-Helix. Energetic differences of per-residue between the WT system and the mutant systems indicated the mutations may allosterically regulate the conformational ensemble and the major variations were residues of Ile-663 and GIn-683, which located in the key loops of catalytic loop and A-loop, respectively. The large conformational and energetic differences were further supported by the GaMD simulations. Overall, these obtained molecular mechanisms will aid rational design of novel Mps1 inhibitors to combat inhibitor resistance. 
1 Molecular mechanism of point mutation-induced Monopolar spindle 1

2 (Mps1/TTK) inhibitor resistance revealed by a comprehensive molecular

3 modeling study

4

5 Yan $\mathrm{Han}^{1, *}$, Yungang $\mathrm{Wu}^{1}$, Yi Xu${ }^{2}$, Wentao $\mathrm{Guo}^{3}$, $\mathrm{Na}_{\text {Zhang }}{ }^{1}$, Xiaoyi Wang ${ }^{1}$

6

$7{ }^{1}$ Department of TCM Orthopedics \& Traumatology, the First Affiliated Hospital of Wenzhou

8 Medical University, Wenzhou 325003, Zhejiang Province, China.

92 Department of Pharmacy, the First Affiliated Hospital of Wenzhou Medical University,

10 Wenzhou 325003, Zhejiang Province, China

$11{ }^{3}$ School of Pharmacy, Wenzhou Medical University, Wenzhou 325003, Zhejiang, China.

12

* Correspondence

14

15

16

17

18

19

20

9

Yan Han

Department of TCM Orthopedics \& Traumatology, the First Affiliated Hospital of Wenzhou Medical University, Nanbaixiang, Ouhai District, Wenzhou 325003, Zhejiang Province, China

E-mail: hanyan@wzhospital.cn

Tel: $+86-0577-88824117$; Fax: $+86-0577-88824117$ 


\section{ABSTRACT}

Background. Monopolar spindle 1 (Mps1/TTK) is an apical dual-specificity protein kinase in the spindle assembly checkpoint (SAC) that guarantees accurate segregation of chromosomes during mitosis. High levels of Mps1 are found in various types of human malignancies, such as glioblastoma, osteosarcoma, hepatocellular carcinoma, and breast cancer. Several potent inhibitors of Mps1 exist, and exhibit promising activity in many cell cultures and xenograft models. However, resistance due to point mutations in the kinase domain of Mps1 limits the therapeutic effects of these inhibitors. Understanding the detailed resistance mechanism induced by Mps1 point mutations is therefore vital for the development of novel inhibitors against malignancies.

Methods. In this study, conventional molecular dynamics (MD) simulation and Gaussian accelerated MD (GaMD) simulation were performed to elucidate the resistance mechanisms of Cpd-5, a potent Mps1 inhibitor, induced by the four representative mutations I531M, I598F, C604Y, S611R.

Results. Our results from conventional MD simulation combined with structural analysis and free energy calculation indicated that the four mutations weaken the binding affinity of Cpd-5 and the major variations in structural were the conformational changes of P-loop, A-loop and $\alpha \mathrm{C}-\mathrm{Helix}$. Energetic differences of per-residue between the WT system and the mutant systems indicated the mutations may allosterically regulate the conformational ensemble and the major variations were residues of Ile-663 and Gln-683, which located in the key loops of catalytic loop and A-loop, respectively. The large conformational and energetic differences were further supported by the GaMD simulations. Overall, these obtained molecular mechanisms will aid rational design of novel Mps 1 inhibitors to combat inhibitor resistance. 


\section{INTRODUCTION}

Mammalian cell division is accurately regulated by activation and inactivation of related proteins that manage progression through the phases of the cell cycle (Bertoli et al. 2013). To ensure segregation of duplicated chromosome during mitosis and meiosis, cell-cycle checkpoints have evolved that play essential roles in genome maintenance under varieties of stress conditions (Barnum \& O'Connell 2014). The spindle assembly checkpoint (SAC), one of cell-cycle checkpoints and a signaling cascade, prevents chromosome missegregation by delaying mitotic progression until all chromosomes are correctly attached to spindle microtubules (Hiruma et al. 2015). Inactivation of SAC leads to premature anaphase onset, and, therefore, chromosomal instability and aneuploidy, which is responsible for cell death or tumorigenesis (de Carcer et al. 2007; Marques et al. 2015; Salmela \& Kallio 2013).

The mono-polar spindle 1 (Mps1, also called TTK), One of the main components of SAC, is generally regarded as a master conductor of SAC signaling, which recruit in early mitosis to unattached kinetochores (Pachis \& Kops 2018). Mps1 has been proposed to be dysregulated in various cancer cells (Xie et al. 2017). For instance, mRNA expression of Mps1 is elevated in numerous of cancers relative to normal tissue. These include glioblastoma, osteosarcoma, hepatocellular carcinoma, breast cancer, and other cancers. Reduction in Mps1 levels or activity in these tumors can lead to loss of cell viability, therefore, inhibition of Mps1 has been regard as an attractive strategy to target cancers, especially those with chromosomal instability (Daniel et al. 2011; Xie et al. 2017). Many structurally diverse Mps1 inhibitors have been reported and have undergone preclinical assessments in recent years, such as NMS-P715, Cpd-5, CCT251455, MPS1-IN-3, reversine, MPI-0479605, AZ3146 (Colombo et al. 2010; Hewitt et al. 2010; Hiruma et al. 2017; Naud et al. 2013; Tannous et al. 2013; Tardif et al. 2011). Unfortunately, in cancer cells, the resistance to Mps1 inhibitors eventually occurs and this resistance is mostly due to mutations in the conserved ATP binding pocket of the Mps1 kinase domain, which leads to a remarkably attenuation of the therapeutic efficiency of the Mps1 inhibitors (Hiruma et al. 2017; 
Koch et al. 2016).

The kinase domain of Mps1 adopts a bi-lobal structure, a smaller N-terminal domain, and a larger C-terminal domain connected by kinase hinge, which together form the catalytic site to ensure the transfer of a phosphate from adenosine triphosphate (i.e., ATP) to a substrate hydroxyl (Figure 1A). The N-terminal domain contains six stranded antiparallel $\beta$-sheets, an important regulatory $\alpha \mathrm{C}$-helix, and a phosphate binding loop (i.e., P-loop). The C-terminal domain consists of seven $\alpha$-helices, two $\beta$-sheets, a catalytic loop, and an essential activation loop (i.e., A-loop, residues 676-685) (Chu et al. 2008; Wang et al. 2009). Recently, Koch et al. (Koch et al. 2016) reported four point mutations (I531M, I598F, C604Y, S611R) in the kinase domain of Mps1 that gave rise to the Cpd-5 resistance, but retained wild type (WT) catalytic activity. Cpd-5 (N-(2,6diethylphenyl)-8-((2-methoxy-4-(4-methylpiperazin-1-yl)phenyl)amino)-1-methyl-4,5-dihydro1H-pyrazolo[4,3h] quinazoline-3 carboxamide), a derivative of NMS-P715, has been reported to display higher potency toward Mps1 than NMS-P715, reversine, MPI-0479605 (Koch et al. 2016). Recently, Chen et al. (Chen et al. 2018) reported resistance mechanisms of inhibitors to Mps1 C604Y Mutation, however, it is not clear from the structures why other mutations (I531M, I598F, S611R) would cause resistance. Molecular dynamics (MD) simulations may be helpful to explain these resistance mutations by monitoring the dynamics of the protein and its interactions with bound inhibitors.

In this study, the WT Mps1 (Mps1 ${ }^{\mathrm{WT}}$ ) and four mutants associated with Cpd-5 resistance, including I531M (Mps 1531M), I598F (Mps1 $\left.{ }^{\mathrm{I598F}}\right)$, C604Y (Mps1 $\left.{ }^{\mathrm{C} 604 \mathrm{Y}}\right)$, S611R (Mps1 $\left.{ }^{\mathrm{S} 611 \mathrm{R}}\right)$, were chosen to create an overall view of the structural dynamics. Herein, conventional molecular dynamics (MD) simulations combined with structural analysis and Molecular Mechanics/Generalized Born Surface Area (MM/GBSA) free energy calculations were employed to elucidate the impact of point mutations on the conformational and energetic difference between WT and mutant systems. In addition, Gaussian accelerated molecular dynamics (GaMD) simulations in conjunction with structural analysis, principal component analysis (PCA) and 2D free energy calculations were performed to sample more conformational space due to conventional 
103

104

105

106

107

108

109

110

111

112

113

114

115

116

117

118

119

120

121

122

123

124

125

126

127

128

129

MD simulation remains limited conformational ensembles. Overall, the comprehensive molecular modeling study performed in this study can be effective to study the resistance mechanism. More importantly, the insights from this study may have an essential application in designing novel Mps1 inhibitors to combat inhibitor resistance.

\section{MATERIALS \& METHODS}

\section{Construct the Initial Structures of Mps1 ${ }^{\mathrm{WT}}$, Mps1 $1^{\mathrm{I531M}}, \mathrm{Mps}^{1598 \mathrm{~F}}$, Mps1 ${ }^{\mathrm{S611R}}$}

The three-dimensional structure of the $\mathrm{Mps} 1^{\mathrm{C} 604 \mathrm{Y}} / \mathrm{Cpd}-5$ complex was retrieved from Protein Data Bank (PDB) database (PDB entry: 5MRB) (Hiruma et al. 2017). Mps1 ${ }^{\mathrm{WT}}, \mathrm{Mps} 1^{\mathrm{I} 31 \mathrm{M}}$, Mps1 ${ }^{\mathrm{I} 998 \mathrm{~F}}$, Mps $1^{\mathrm{S} 611 \mathrm{R}}$ were constructed by using EasyModeller program by substituting specific residues (Fiser \& Sali 2003; Kuntal et al. 2010). Then, the modeled structures were refined by Chimera software, including modelling the A-loop, adding missing side-chains and missing hydrogen atoms (Pettersen et al. 2004).

\section{Conventional molecular dynamics (MD) simulations}

The crystal structure of $\mathrm{Mps} 1^{\mathrm{C} 604 \mathrm{Y}} / \mathrm{Cpd}-5$, and the modeled structures of $\mathrm{Mps} 1^{\mathrm{WT}} / \mathrm{Cpd}-5$, Mps 1 $1531 \mathrm{M} / \mathrm{Cpd}-5, \mathrm{Mps} 1^{1598 \mathrm{~F}} / \mathrm{Cpd}-5, \mathrm{Mps} 1^{\mathrm{S} 611 \mathrm{R}} / \mathrm{Cpd}-5$ were used as the initial structures for the conventional MD simulations. Prior to conventional MD simulations, the force field parameters for the proteins and ligands were generated using LEaP module in Amber 16 software with AMBER ff14SB force field and general Amber force field 2 (GAFF2). Thereafter, each system was immersed in a water box by the TIP3P water model with at least a $12 \AA$ distance around the protein-ligand complex. Finally, an appropriate number of counter ions were added to keep the electroneutrality.

Before each productive MD simulation, a sophisticated protocol was applied, including minimization, heating and equilibration. The first step of energy minimization was to employed a harmonic restraint of $5 \mathrm{kcal} \mathrm{mol}^{-1} \AA^{-2}$ to the solute and side chains of protein. The second step was to allow all atoms to move freely without any restraint. In each step, minimization was performed 
130

131

132

133

134

135

136

137

138

139

140

141

142

143

144

145

146

$$
\begin{aligned}
& \Delta G_{\text {calc }}=\Delta \mathrm{E}_{\mathrm{MM}}+\Delta \mathrm{G}_{\text {sol }}-\mathrm{T} \Delta \mathrm{S} \\
& \Delta \mathrm{E}_{\mathrm{MM}}=\Delta \mathrm{E}_{\text {int }}+\Delta \mathrm{E}_{\mathrm{vdW}}+\Delta \mathrm{E}_{\text {elec }} \\
& \Delta \mathrm{G}_{\mathrm{sol}}=\Delta \mathrm{G}_{\mathrm{GB}}+\Delta \mathrm{G}_{\mathrm{SA}} \\
& \Delta \mathrm{G}_{\mathrm{SA}}=\gamma \Delta \mathrm{A}+\mathrm{b}
\end{aligned}
$$

147

Where $\Delta E_{\mathrm{MM}}, \Delta G_{\mathrm{sol}}$, and $-\mathrm{T} \Delta \mathrm{S}$ represent the molecular mechanics interaction energy, solvation energy, and entropy term (equation 1). In equation (2), the $\Delta E_{\mathrm{MM}}$ consists of the change of internal energy $\left(\Delta E_{\text {int }}\right)$, van der Waals energy $\left(\Delta E_{\mathrm{vdW}}\right)$, and electrostatic energy $\left(\Delta E_{\text {elec }}\right)$. In equation (3), the $\Delta G_{\mathrm{sol}}$ contains the polar part $\left(\Delta G_{\mathrm{GB}}\right)$ and the nonpolar part of the desolvation energy $\left(\Delta G_{S A}\right)$. In this study, $\Delta E_{\text {int }}$ was canceled by using a single trajectory strategy in order to reduce the noise (Sun et al. 2013). The $\Delta G_{\mathrm{GB}}$ was calculated using a $\mathrm{GB}^{\mathrm{OBC} 1}$ model (igb $=2$ ) (Onufriev et al. 2000). The $\Delta G_{S A}$ was calculated by equation (4). Where $\Delta A$ is the change of the 
154

155

156

157

158

159

160

161

162

164

165

166

167

$$
\begin{array}{ll}
V^{*}(\vec{r})=V(\vec{r})+\frac{1}{2} \mathrm{k}(E-V(\vec{r}))^{2} & V(\vec{r})<E \\
V^{*}(\vec{r})=V(\vec{r}) & V(\vec{r}) \geq E
\end{array}
$$

\section{Gaussian accelerated molecular dynamics (GaMD) simulation}

The equilibrated structures extracted from conventional MD simulation trajectories were chosen as the initial structure for the GaMD simulations. During GaMD simulations, a non-negative harmonic boost potential is added to smooth the studied system potential energy surface in order to decrease the energy barriers and accelerate the conformational sampling (Miao \& McCammon 2016a; Miao \& McCammon 2016b). When the system potential $V(\vec{r})$ is lower than a threshold energy $(E)$, a harmonic boost potential $V^{*}(\vec{r})$ is added as equation (5). $k$ is the harmonic force constant. When the system potential is above the $E$, the boost potential is set to zero as equation (6).

168

GaMD simulation provides the total potential boost, dihedral potential boost, and dual potential boost in order to accelerate the MD simulations. Herein, dual potential boost was applied to the GaMD simulations. The dual potential boost parameters were computed from an initial $\sim 4$ ns NVT conventional MD simulation without any potential boost. Afterwards, 1 ns GaMD simulation were performed, in which the potential boost was updated every 100 ps to reach equilibrium values. Eventually, $400 \mathrm{~ns}$ GaMD simulation for each system was submitted in the NVT ensemble. During the simulations, the temperature was regulated using a Langein thermostat. PME algorithm and the non-bonded with cutoff $10.0 \AA$ to consider the long-range electrostatic interactions and non-bonding interactions (Essmann et al. 1995). Atomic coordinate trajectory was recorded every 2 ps. 
Principal component analysis (PCA) and free energy calculations for the GaMD simulations

181

182

183

184

185

186

187

188

PCA calculations contain diagonalization of the covariance matrix of positional deviations among a structural ensemble. The structures from MD simulation trajectories were aligned to remove the translational and rotational motions. PCA was performed for the trajectories of $400 \mathrm{~ns}$ GaMD simulations by using Bio3D package of R (Skjaerven et al. 2014). Thereafter, the potential boost combined with the principal component $1(\mathrm{PC} 1)$ and principal component 2 (PC2) calculated from PCA were applied to recover the free energy map by cumulant expansion to the 2 nd order method (Miao et al. 2014; Roe \& Cheatham 2013).

\section{RESULTS and DISCUSSION}

\section{The overall structural properties}

The structural dynamics of Cpd-5 bound with WT Mps1 or mutant Mps1 were analyzed by performing $240 \mathrm{~ns}$ conventional MD simulations. To qualitatively investigate the stability and overall convergence of the simulated systems, the root mean square deviation (RMSD) of protein backbone atoms, and the heavy atoms of the Cpd-5 were calculated and plotted against time in Figure 2 and 3. As plotted in Figure 2, all the RMSD values of backbone atoms of Mps1 have a small fluctuation after 50-170 ns, indicating all the systems achieve equilibrium at $\sim 50-170 \mathrm{~ns}$. The RMSD of the heavy atoms of the Cpd-5 in each system maintained relative stable (RMSD < $2 \AA$ ) during the $240 \mathrm{~ns}$ simulation (Figure 3). As expected, the average RMSD values of both the Mps 1 protein and the Cpd-5 follow the order of WT systems < mutant systems (Figure 2 and 3 ). These findings are directly consistent with the experimental data that Cpd-5 is more stable in WT Mps1 than in the mutants. Thereafter, the trajectories of the last $60 \mathrm{~ns}$ simulations were extracted for the following structural and energetic analysis.

Then, the root-mean-square fluctuations (RMSFs) of the protein backbone, which represents the flexibility and mobility of protein backbone, were calculated and averaged for all the simulated systems to evaluate the local structure transformations in more detail. As shown in Figure 4, all 
206 the simulated systems share similar RMSF distributions and similar trends of dynamics features.

207 However, we noticed P-loop region of Mps1531M, Mps1 $1^{1598 F}$, Mps $1^{\mathrm{C} 604 \mathrm{Y}}$, $\alpha \mathrm{C}$-helix region of

$208 \mathrm{Mps} 1^{1598 \mathrm{~F}}$, A-loop region of all the four mutant systems, which exhibited amplified fluctuations

209 when these regions compared to WT system. The alignment of the last snapshot between WT

210 (yellow) system and mutant (cyan) systems showed a highly similar pattern with minor

211 adjustments in the regions of P-loop, $\alpha \mathrm{C}$-helix, A-loop (Figure 5). For instance, compared with

$212 \mathrm{Mps} 1^{\mathrm{WT}}$, the P-loop region of $\mathrm{Mps} 1^{\mathrm{I} 331 \mathrm{M}}, \mathrm{Mps} 1^{\mathrm{I598F}}, \mathrm{Mps} 1^{\mathrm{C} 604 \mathrm{Y}}$ and the $\alpha \mathrm{C}$-helix region of

$213 \mathrm{Mps} 1^{\mathrm{I5} 98 \mathrm{~F}}$ change into upward-moving conformation compared with Mps $1^{\mathrm{WT}}$ (Figure 5B-5D). The

214 A-loop region of mutant systems showed an outward-moving conformation (Figure 5A-5D). This

215 finding was also consistent with the previous study that when Mps1 binds with Cpd-5, the A-loop

216 region of $\mathrm{Mps}^{\mathrm{C} 604 \mathrm{Y}}$ changes into an outward-moving conformation, but not the Mps1 WT (Chen et

217 al. 2018). Compared with the previous results, we also found that P-loop had a slight 218 conformational change (Chen et al. 2018), particularly in Mps $1^{\mathrm{I} 598 \mathrm{~F}}$ and Mps1 ${ }^{\mathrm{C} 604 \mathrm{Y}}$ systems.

219 Overall, these results indicated that mutation-induced conformational change might be the main

220 driving force for the redistributed energies. Hence, the MM/GBSA approach was employed to

221 determine the key molecular determinants.

\section{Binding free energy and decomposition analysis}

224

225

226

227

228

As an important complement to the binding mode and structural analysis discussed above, the binding free energies of the Cpd-5 to the WT system and the mutant systems calculated from the MM-GBSA approach were summarized in Table 1. According to Table 1, the predicted binding free energies $\left(\Delta G_{\text {calc }}\right)$ of Cpd-5 to Mps1 ${ }^{\mathrm{WT}}, \mathrm{Mps}^{\mathrm{I531M}}, \mathrm{Mps} 1^{\mathrm{I598F}}, \mathrm{Mps}^{\mathrm{C} 604 \mathrm{Y}}, \mathrm{Mps} 1^{\mathrm{S} 611 \mathrm{R}}$ were $59.73 \pm 4.72,-51.89 \pm 4.14,-52.00 \pm 4.61,-49.77 \pm 3.67$, and $-54.66 \pm 3.74 \mathrm{kcal} / \mathrm{mol}$, respectively. It can be observed that the $\Delta G_{\text {calc }}$ follow the order of WT systems $<$ mutant systems, that is to say, the $\Delta G_{\text {calc }}$ show a high correlation with the reported experimental data of $\mathrm{IC}_{50}$. Then, individual components of binding free energy were analyzed, which could provide useful information to understand the inhibitor resistance mechanism followed by individual contribution 
233

234

235

236

237

238

239

240

241

242

243

244

245

246

247

248

249

250

251

252

253

254

255

256

257

258

259

260

decompositions to the binding free energies. As shown in Table 1, the van der Waals contributions of Cpd-5 to Mps1 ${ }^{\mathrm{WT}}, \mathrm{Mps} 1^{\mathrm{I} 31 \mathrm{M}}, \mathrm{Mps} 1^{\mathrm{I598F}}, \mathrm{Mps} 1^{\mathrm{C} 604 \mathrm{Y}}, \mathrm{Mps} 1^{\mathrm{S} 611 \mathrm{R}}$ were $-73.57 \pm 4.62,-62.15 \pm 3.78$, $-63.28 \pm 5.09,-61.16 \pm 3.89,-66.43 \pm 4.28 \mathrm{kcal} / \mathrm{mol}$, respectively, which determine the difference of the binding free energies between WT and mutant systems.

To further probe the interaction between Cpd-5 and Mps1, per-residue decomposition analysis was conducted to obtain a more detailed description of each residue contribution to the binding free energy by MM/GBSA decomposition approach. Energetic differences of per-residue between the WT system and the mutant systems $\left(\Delta \Delta \mathrm{G}=\Delta \mathrm{G}_{\mathrm{WT}}-\Delta \mathrm{G}_{\text {mutant }}\right)$ were plotted to highlight the key residues, which induced redistribution of binding free energy. As shown in Figure 6, the negative values indicated that the residues of the WT Mps1 protein form stronger interactions with Cpd-5 than the mutant Mps1 protein, while the positive values suggested that the residues of the mutant Mps1 protein form stronger interactions with Cpd-5 than the WT Mps1 protein. As plotted in Figure 6A, the key residues of Ile-663 and Gln-683 have significant stronger interactions to Cpd5 in the WT system than in all the mutant systems. Notably, the residues of Ile-663 and Gln-683 are located in the two flexible key loops of catalytic loop and A-loop, respectively, which are important in rotating the Asp-Phe-Gly (DFG) motif into proper orientation for catalysis and substrate binding (Figure 6B and 6C). These observations may helpful to aid rational design of novel Mps1 inhibitors to overcome inhibitor resistance. For instance, moderately increasing the hydrophobicity of the compound to interact with Ile-663 to stabilize the catalytic loop and forming hydrogen bonds with Gln-683 to stabilize the A-loop.

\section{GaMD simulations}

All-atom conventional MD simulations are still limited to the conformational ensembles because of the possible energy barriers between various intermediate states. Therefore, an enhanced sampling method, which can take samples at various intermediate states is required. The traditional enhanced sampling often requires predefined parameters, such as root-mean-square distance atom distances, torsional dihedral, which usually needs expert knowledge of the studied systems. However, the enhanced sampling technique of GaMD simulation avoids such a requirement. In 
261

262

263

264

265

266

267

268

269

270

271

272

273

274

275

276

277

278

279

280

281

282

283

284

285

286

287

this study, we applied the GaMD simulation technique to further explore more conformational states. Following the initial $240 \mathrm{~ns}$ conventional MD simulation, the last snapshot of each system was used as the initial structure to GaMD simulation.

The RMSDs of the protein backbone and the heavy atoms of Cpd-5 with respect to the starting conformations were calculated after alignment of all the conformations from GaMD simulation by removing the rotational and translational motions (Figure 7 and 8). As shown in Figure 7, the RMSD of protein backbones of all the systems achieved equilibrium after 30-200 ns GaMD simulations. Figure 8 showed the RMSDs of the heavy atoms of Cpd-5 in each system maintained dynamic constant during the $400 \mathrm{~ns}$ of GaMD simulation, indicating the stability of the Cpd-5 in the studied systems.

In addition, the average RMSD values of both the protein and the ligand follow the order of WT systems $<$ mutant systems, which are similar with the results from the conventional MD simulation. The above observations suggested that these four point mutations allowed larger conformational changes and more variability among protein subunits. Then, the last snapshots of each mutant system (magenta) extracted from GaMD simulation trajectories were superimposed with the last snapshot of WT system (green) to obtain an intuitionistic description of the conformational changes among these systems. As shown in Figure 9A-9D, most conformational changes occurred in P-loop and A-loop. These findings also supported the observation from conventional MD simulations. Compared with $\mathrm{Mps} 1^{\mathrm{WT}}$, the P-loop of Mps ${ }^{\mathrm{I5} 98 \mathrm{~F}}, \mathrm{Mps} 1^{\mathrm{C} 604 \mathrm{Y}}$, and A-loop of all the mutant systems changed into upward-moving conformations and outwardmoving conformations, respectively.

PCA was performed to further characterize the conformational transitions and plotted against time (Figure 10). PCA reduces the dimensionality of large data sets and the vectors with the highest eigenvalues represent the most significant principal components (PCs). When principal components are plotted against each other, similar structures are clustered. Theoretically, each cluster shows a different protein conformational state. As shown in Figure 10 and Figure S1, the conformational states of both the WT and mutant systems were dynamics and functions during 0- 
288

289

290

291

292

400 ns GaMD simulations, and finally, stabilized in one state. Compared to the WT system, the mutant systems exhibited more structural clusters and larger eigenvalues. These conformer plots highlighted the major differences between WT and mutant systems, which interpreted the observations of large conformational changes when resistance mutations occurred by conventional MD simulations and GaMD simulations.

The free energy landscape was utilized to further explain the relationship between the conformational change and energetic change. More energetic wells (dark blue regions) represent the protein underwent larger conformational change. As shown in Figure 11, the WT system was confined to a major energetic deep well throughout the simulation (Figure 11A), while numerous of energetic deep wells were observed for the four mutant systems (Figure 11B-11F), highlighting an ensemble of different conformational states distributed over a large free energy space. Additionally, the free energy landscape between the four mutant systems also showed significant difference (Figure 11B-11F), suggesting the four mutations may allosterically regulate the conformational ensemble to induce inhibitors resistance.

\section{CONCLUSIONS}

In this study, molecular modeling, conventional MD simulations and GaMD simulations successfully clarified the resistance mechanism induced by the four point mutations of I531M, I598F, C604Y, S611R, both structurally and energetically. The binding free energies of Cpd-5 to the WT system and mutant systems were well predicted and the decomposition of the individual energy terms suggested the major variation of Cpd-5 between the WT system and the mutant systems were van der Waals interactions. Structural analysis revealed the conformational changes of P-loop, A-loop and $\alpha \mathrm{C}$-helix play crucial role for $\mathrm{Cpd}-5$ resistance. Further energetic differences of per-residue between the WT system and the mutant systems revealed the four mutations may allosterically regulate the conformational ensemble and the major variations were residues of Ile663 and Gln-683, which located in the key loops of catalytic loop and A-loop, respectively. In addition, GaMD simulations supported the observations from conventional MD simulations. PCA 
315

316

317

318

319

320

321

322

323

324

325

326

327

328

329

330

331

332

333

334

335

336

337

338

339

340

341

342

343

344

345

346

347

348

349

350

351

352

results and free energy landscape from GaMD simulations indicated Mps1 underwent large conformational changes when the resistance mutations occurred. In summary, our study not only revealed the resistance determinants of $\mathrm{Cpd}-5$ to the four point mutations, but also provide some valuable information for structure based design of novel inhibitors of Mps1 in the future.

\section{REFERENCES}

Barnum KJ, and O'Connell MJ. 2014. Cell cycle regulation by checkpoints. Methods Mol Biol 1170:29-40. 10.1007/978-1-4939-0888-2_2

Bertoli C, Skotheim JM, and de Bruin RA. 2013. Control of cell cycle transcription during G1 and S phases. Nat Rev Mol Cell Biol 14:518-528. 10.1038/nrm3629

Chen Y, Yu W, Jiang CC, and Zheng JG. 2018. Insights into Resistance Mechanisms of Inhibitors to Mps1 C604Y Mutation via a Comprehensive Molecular Modeling Study. Molecules 23. 10.3390/molecules23061488

Chu ML, Chavas LM, Douglas KT, Eyers PA, and Tabernero L. 2008. Crystal structure of the catalytic domain of the mitotic checkpoint kinase Mps1 in complex with SP600125. J Biol Chem 283:21495-21500. 10.1074/jbc.M803026200

Colombo R, Caldarelli M, Mennecozzi M, Giorgini ML, Sola F, Cappella P, Perrera C, Depaolini SR, Rusconi L, Cucchi U, Avanzi N, Bertrand JA, Bossi RT, Pesenti E, Galvani A, Isacchi A, Colotta F, Donati D, and Moll J. 2010. Targeting the mitotic checkpoint for cancer therapy with NMS-P715, an inhibitor of MPS1 kinase. Cancer Res 70:10255-10264. 10.1158/0008-5472.CAN-10-2101

Daniel J, Coulter J, Woo JH, Wilsbach K, and Gabrielson E. 2011. High levels of the Mps1 checkpoint protein are protective of aneuploidy in breast cancer cells. Proc Natl Acad Sci U S A 108:5384-5389. 10.1073/pnas. 1007645108

de Carcer G, Perez de Castro I, and Malumbres M. 2007. Targeting cell cycle kinases for cancer therapy. Curr Med Chem 14:969-985.

Essmann U, Perera L, Berkowitz ML, Darden T, Lee H, and Pedersen LG. 1995. A smooth particle mesh Ewald method. The Journal of chemical physics 103:8577-8593.

Fiser A, and Sali A. 2003. Modeller: generation and refinement of homology-based protein structure models. Methods Enzymol 374:461-491. 10.1016/S0076-6879(03)74020-8

He M, Li W, Zheng Q, and Zhang H. 2018. A molecular dynamics investigation into the mechanisms of alectinib resistance of three ALK mutants. J Cell Biochem 119:5332-5342. 10.1002/jcb.26666

Hewitt L, Tighe A, Santaguida S, White AM, Jones CD, Musacchio A, Green S, and Taylor SS. 2010. Sustained Mps1 activity is required in mitosis to recruit O-Mad2 to the Mad1-C-Mad2 core complex. J Cell Biol 190:25-34. 10.1083/jcb.201002133

Hiruma Y, Koch A, Hazraty N, Tsakou F, Medema RH, Joosten RP, and Perrakis A. 2017. Understanding inhibitor resistance in Mps1 kinase through novel biophysical assays and structures. $J$ Biol Chem 292:14496-14504. 10.1074/jbc.M117.783555

Hiruma Y, Sacristan C, Pachis ST, Adamopoulos A, Kuijt T, Ubbink M, von Castelmur E, Perrakis A, and Kops 
GJ. 2015. CELL DIVISION CYCLE. Competition between MPS1 and microtubules at kinetochores regulates spindle checkpoint signaling. Science 348:1264-1267. 10.1126/science.aaa4055

Izaguirre JA, Catarello DP, Wozniak JM, and Skeel RD. 2001. Langevin stabilization of molecular dynamics. The Journal of chemical physics 114:2090-2098.

Koch A, Maia A, Janssen A, and Medema RH. 2016. Molecular basis underlying resistance to Mps1/TTK inhibitors. Oncogene 35:2518-2528. 10.1038/onc.2015.319

Kräutler V, Van Gunsteren WF, and Hünenberger PH. 2001. A fast SHAKE algorithm to solve distance constraint equations for small molecules in molecular dynamics simulations. Journal of computational chemistry 22:501-508.

Kuntal BK, Aparoy P, and Reddanna P. 2010. EasyModeller: A graphical interface to MODELLER. BMC Res Notes 3:226. 10.1186/1756-0500-3-226

Liu HL, Zhong HY, Song TQ, and Li JZ. 2017. A Molecular Modeling Study of the Hydroxyflutamide Resistance Mechanism Induced by Androgen Receptor Mutations. Int $J$ Mol Sci 18 . 10.3390/ijms 18091823

Marques S, Fonseca J, Silva PM, and Bousbaa H. 2015. Targeting the spindle assembly checkpoint for breast cancer treatment. Curr Cancer Drug Targets 15:272-281.

Miao Y, and McCammon JA. 2016a. Graded activation and free energy landscapes of a muscarinic G-proteincoupled receptor. Proc Natl Acad Sci U S A 113:12162-12167. 10.1073/pnas.1614538113

Miao Y, and McCammon JA. 2016b. Unconstrained Enhanced Sampling for Free Energy Calculations of Biomolecules: A Review. Mol Simul 42:1046-1055. 10.1080/08927022.2015.1121541

Miao Y, Sinko W, Pierce L, Bucher D, Walker RC, and McCammon JA. 2014. Improved Reweighting of Accelerated Molecular Dynamics Simulations for Free Energy Calculation. $J$ Chem Theory Comput 10:2677-2689. 10.1021/ct500090q

Naud S, Westwood IM, Faisal A, Sheldrake P, Bavetsias V, Atrash B, Cheung KM, Liu M, Hayes A, Schmitt J, Wood A, Choi V, Boxall K, Mak G, Gurden M, Valenti M, de Haven Brandon A, Henley A, Baker R, McAndrew C, Matijssen B, Burke R, Hoelder S, Eccles SA, Raynaud FI, Linardopoulos S, van Montfort RL, and Blagg J. 2013. Structure-based design of orally bioavailable 1H-pyrrolo[3,2-c]pyridine inhibitors of mitotic kinase monopolar spindle 1 (MPS1). J Med Chem 56:10045-10065. $10.1021 / \mathrm{jm} 401395 \mathrm{~s}$

Onufriev A, Bashford D, and David A. 2000. Modification of the generalized Born model suitable for macromolecules. J Phys Chem 104:3712-3720.

Pachis ST, and Kops G. 2018. Leader of the SAC: molecular mechanisms of Mps1/TTK regulation in mitosis. Open Biol 8. 10.1098/rsob.180109

Pan P, Li Y, Yu H, Sun H, and Hou T. 2013. Molecular principle of topotecan resistance by topoisomerase I mutations through molecular modeling approaches. JChem Inf Model 53:997-1006. 10.1021/ci400066x

Pettersen EF, Goddard TD, Huang CC, Couch GS, Greenblatt DM, Meng EC, and Ferrin TE. 2004. UCSF Chimera--a visualization system for exploratory research and analysis. J Comput Chem 25:1605-1612. $10.1002 /$ jcc. 20084

Roe DR, and Cheatham TE, 3rd. 2013. PTRAJ and CPPTRAJ: Software for Processing and Analysis of Molecular Dynamics Trajectory Data. J Chem Theory Comput 9:3084-3095. 10.1021/ct400341p

Salmela AL, and Kallio MJ. 2013. Mitosis as an anti-cancer drug target. Chromosoma 122:431-449. 
10.1007/s00412-013-0419-8

Skjaerven L, Yao XQ, Scarabelli G, and Grant BJ. 2014. Integrating protein structural dynamics and evolutionary analysis with Bio3D. BMC Bioinformatics 15:399. 10.1186/s12859-014-0399-6

Sun H, Li Y, Li D, and Hou T. 2013. Insight into crizotinib resistance mechanisms caused by three mutations in ALK tyrosine kinase using free energy calculation approaches. J Chem Inf Model 53:2376-2389. $10.1021 / \mathrm{ci} 400188 \mathrm{q}$

Tannous BA, Kerami M, Van der Stoop PM, Kwiatkowski N, Wang J, Zhou W, Kessler AF, Lewandrowski G, Hiddingh L, Sol N, Lagerweij T, Wedekind L, Niers JM, Barazas M, Nilsson RJ, Geerts D, De Witt Hamer PC, Hagemann C, Vandertop WP, Van Tellingen O, Noske DP, Gray NS, and Wurdinger T. 2013. Effects of the selective MPS1 inhibitor MPS1-IN-3 on glioblastoma sensitivity to antimitotic drugs. J Natl Cancer Inst 105:1322-1331. 10.1093/jnci/djt168

Tardif KD, Rogers A, Cassiano J, Roth BL, Cimbora DM, McKinnon R, Peterson A, Douce TB, Robinson R, Dorweiler I, Davis T, Hess MA, Ostanin K, Papac DI, Baichwal V, McAlexander I, Willardsen JA, Saunders M, Christophe H, Kumar DV, Wettstein DA, Carlson RO, and Williams BL. 2011. Characterization of the cellular and antitumor effects of MPI-0479605, a small-molecule inhibitor of the mitotic kinase Mps1. Mol Cancer Ther 10:2267-2275. 10.1158/1535-7163.MCT-11-0453

Wang W, Yang Y, Gao Y, Xu Q, Wang F, Zhu S, Old W, Resing K, Ahn N, Lei M, and Liu X. 2009. Structural and mechanistic insights into Mps1 kinase activation. J Cell Mol Med 13:1679-1694. 10.1111/j.15824934.2008.00605.x

Xie Y, Wang A, Lin J, Wu L, Zhang H, Yang X, Wan X, Miao R, Sang X, and Zhao H. 2017. Mps1/TTK: a novel target and biomarker for cancer. J Drug Target 25:112-118. 10.1080/1061186X.2016.1258568 
Figure 1

Overview of the kinase domain of Mps1 and point mutations.

(A) Overview the structure of the Mps1 kinase domain. The point mutations of I531M, I598F, C604Y, S611R are colored magenta balls. (B) Two-dimensional structure of Cpd-5.

A

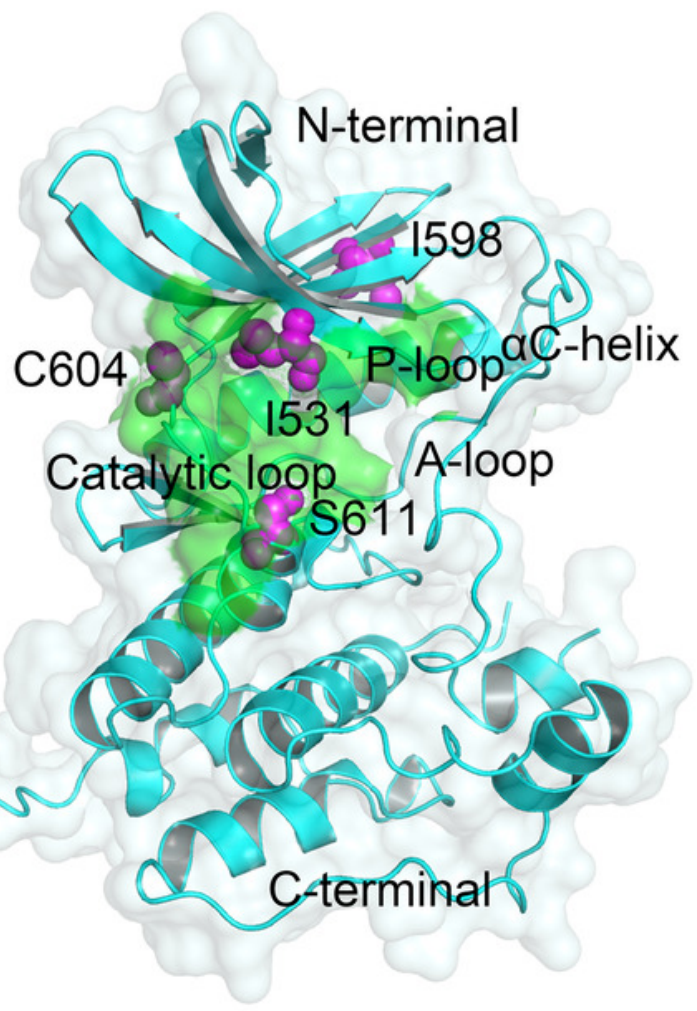

B<smiles>CCc1cccc(CC)c1NC(=O)c1nn(C)c2c1CCC1C=NC(Nc3ccc(N4CCN(C)CC4)cc3OC)=NC21</smiles>

Acquired mutations

$1531 \mathrm{M}$

I598F

C604Y

S611R 
Figure 2

Time evolution of the RMSD values of backbone atoms of the Mps1 protein in the five studied systems from conventional MD simulations. The values reflect the equilibration of each protein relative to the initial structures.

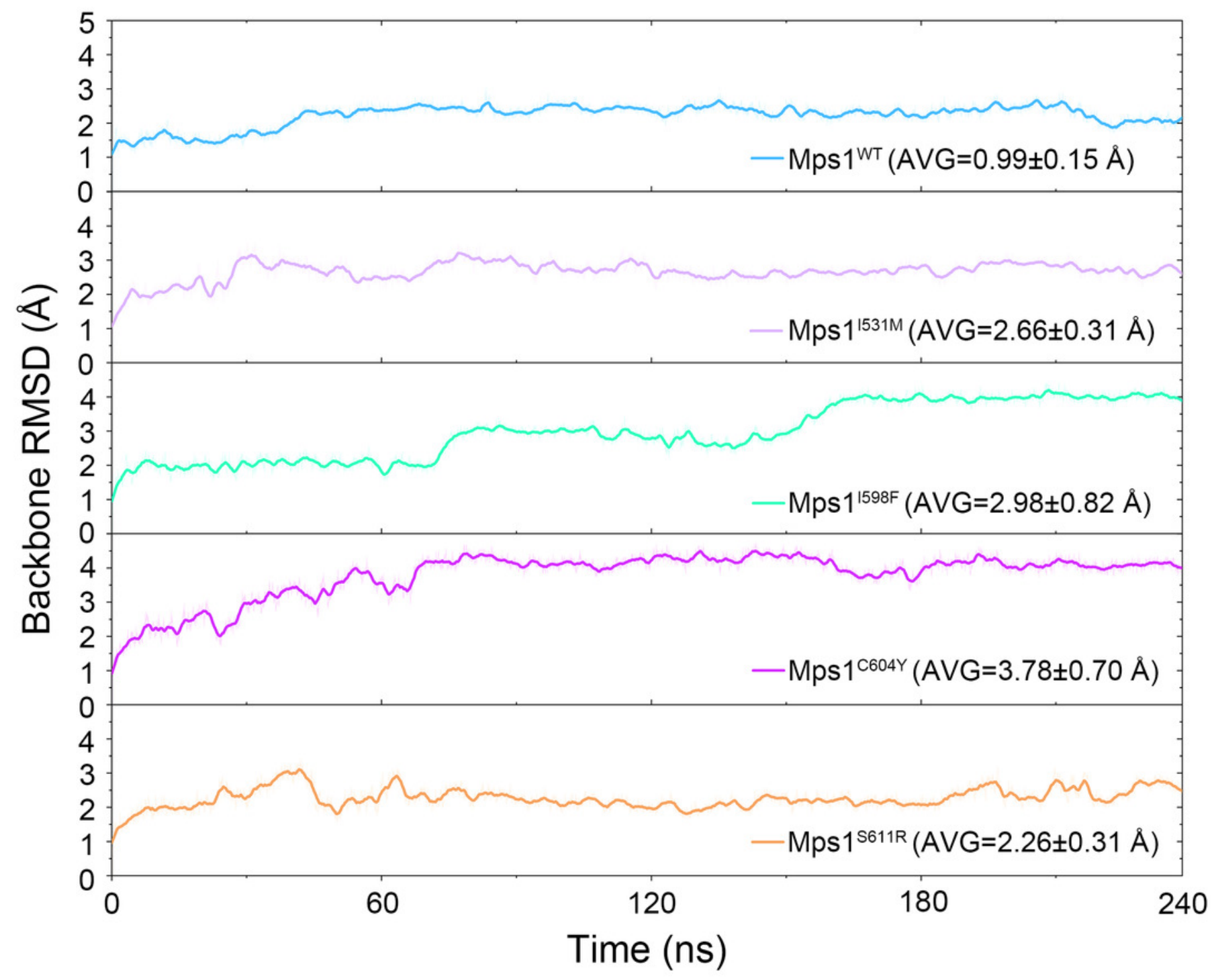


Figure 3

Time evolution of the RMSD values of cpd-5 in the five studied systems from conventional MD simulations. The values reflect the equilibration of Cpd-5 in different protein relative to the initial structures.

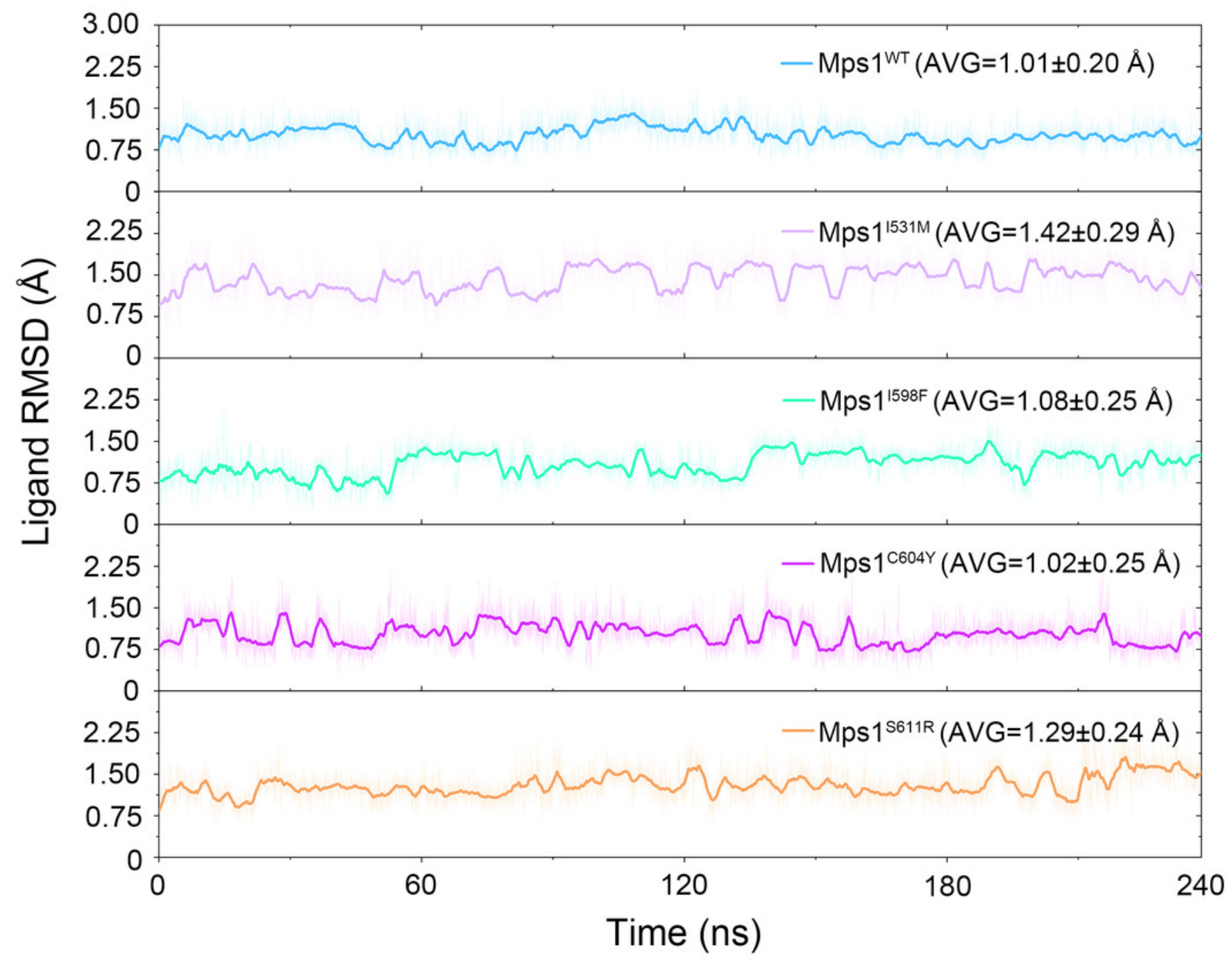


Figure 4

RMSFs of backbone atoms versus residue number in the five studied systems from conventional MD simulations. RMSF provides a picture of overall movement of a residue within a reference frame. Higher RMSF value represent larger conformational change in spec

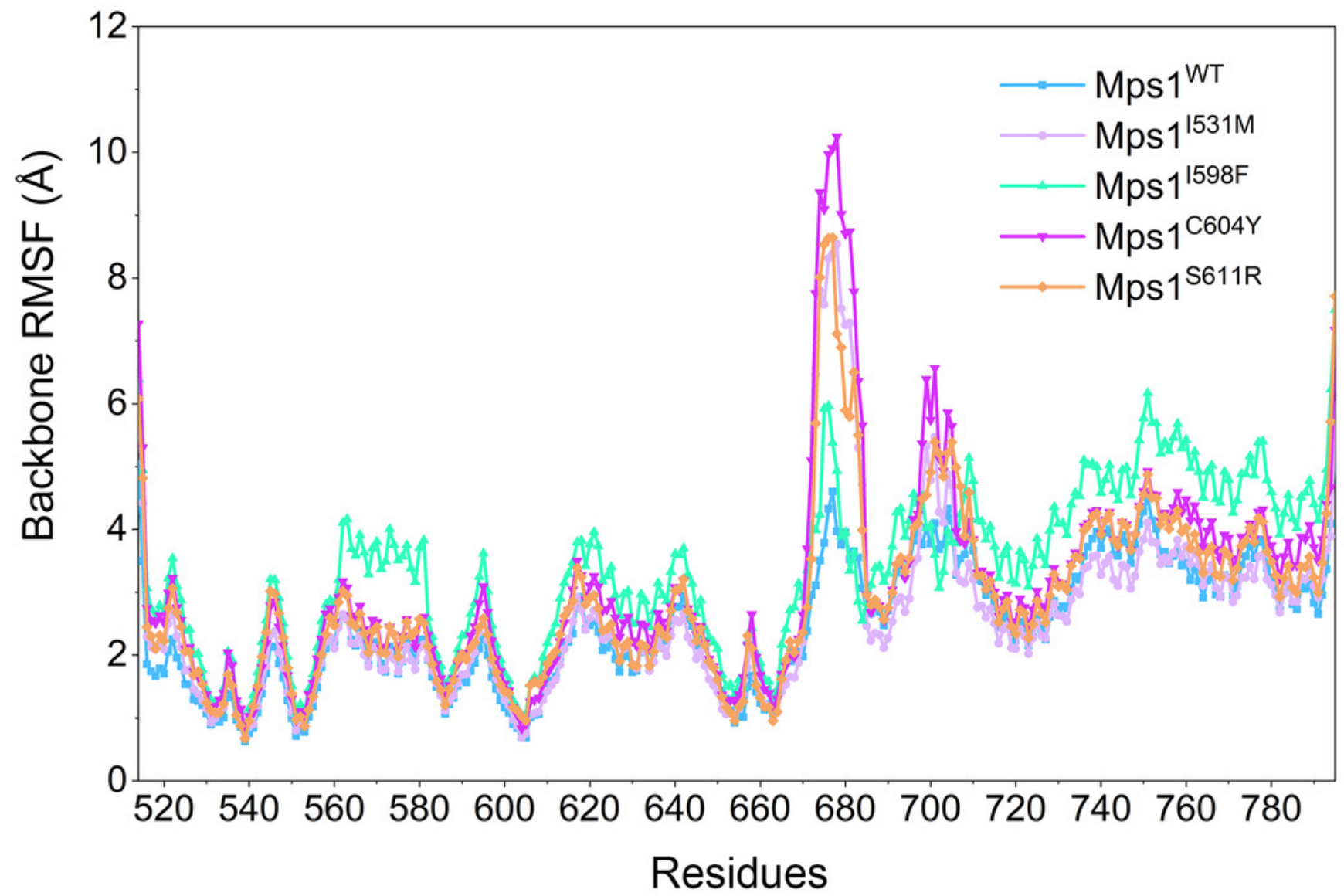


Figure 5

Alignment of the last snapshot between Mps $1^{\mathrm{WT}} / \mathrm{cpd}-5$ and the mutant systems from conventional MD simulations.

(A) Mps1 ${ }^{\mathrm{WT}} / \mathrm{cpd}-5$ (cyan) and Mps1531M/Cpd-5 (yellow). (B) Mps1 ${ }^{\mathrm{WT}} / \mathrm{cpd}-5$ (cyan) and Mps1 $1^{1598 F / C p d-5 ~(y e l l o w) . ~(C) ~ M p s 1 ~}{ }^{\mathrm{WT}} / \mathrm{Cpd}-5$ (cyan) and Mps1 ${ }^{\mathrm{C} 604 \mathrm{Y}} / \mathrm{Cpd}-5$ (yellow). (D) $M p s 1^{\mathrm{WT}} / \mathrm{cpd}-5$ (cyan) and Mps1 $1^{\mathrm{S} 611 R} / \mathrm{Cpd}-5$ (yellow).

A

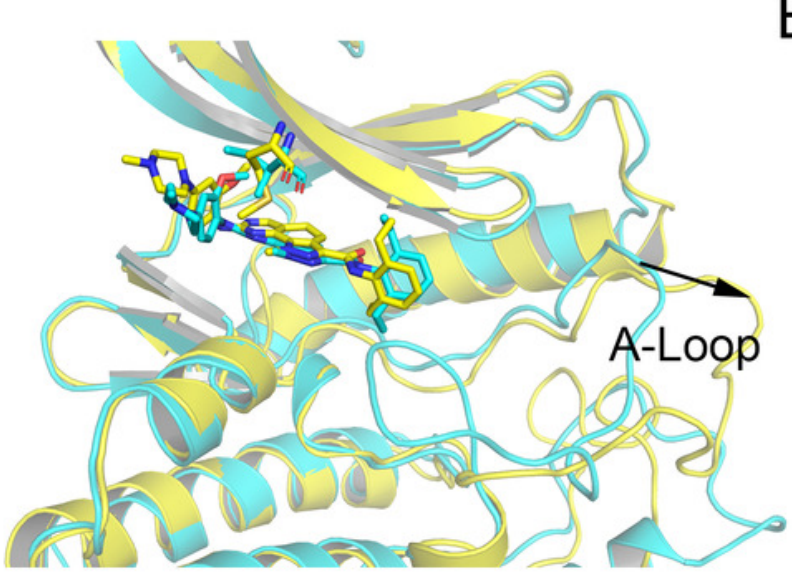

C

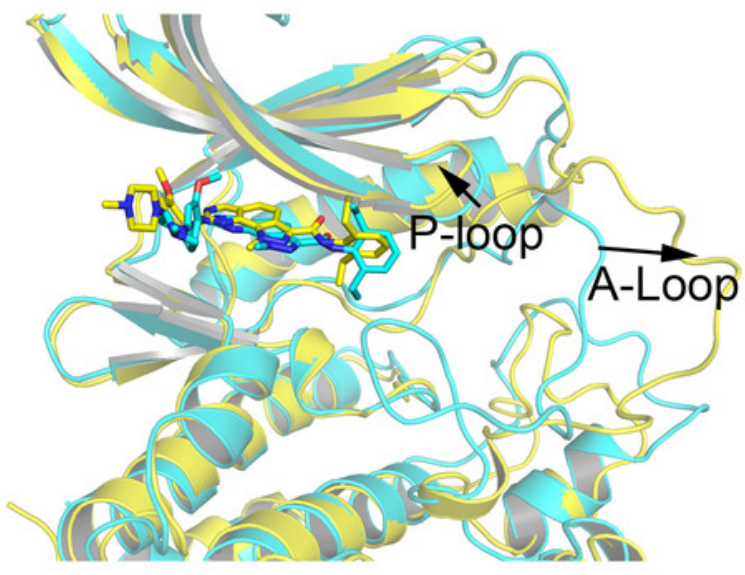

B

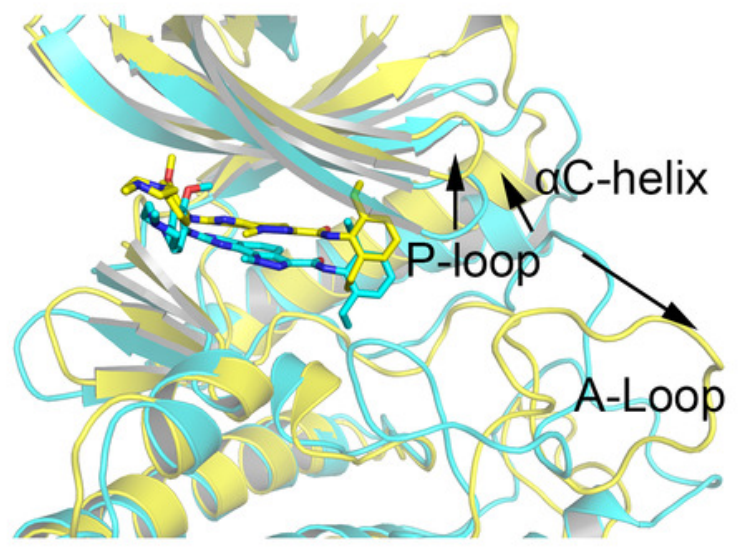

D

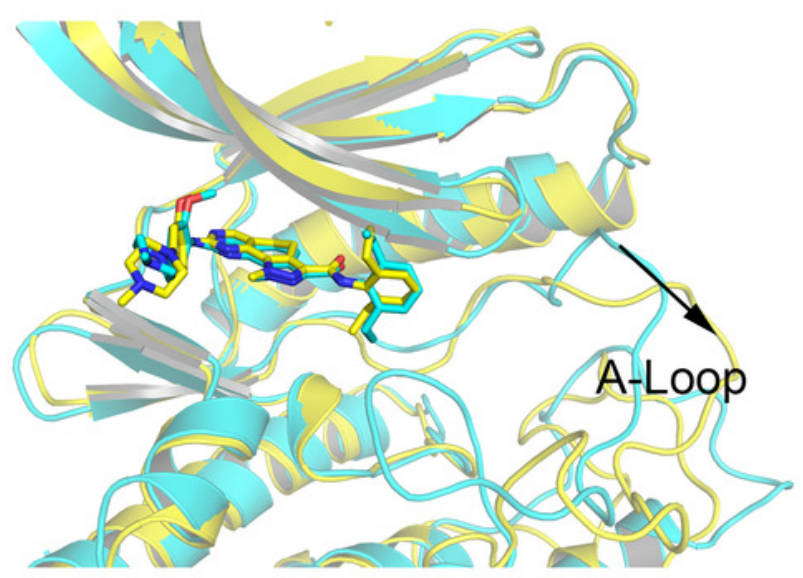


Figure 6

Differences of each residue contribution between the WT system and the mutant systems.

(A) The energetic differences between the WT system and the mutant systems of each residue contribution to Cpd-5 binding; (B) Schematic view of the key residues; (C) Detailed view of the key residues. (C) Detailed view of the key residues.

A



B

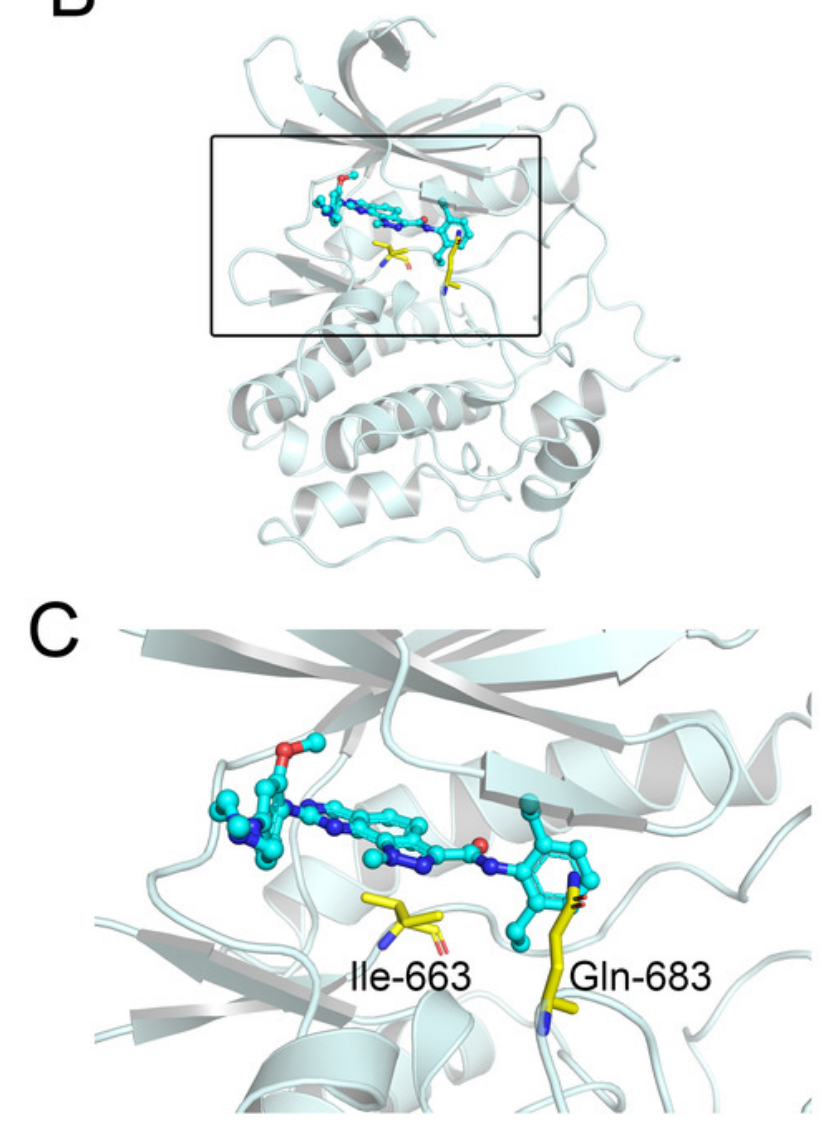


Figure 7

Time evolution of the RMSD values of backbone atoms of the Mps1 protein in the five studied systems from GaMD simulations. The values reflect the equilibration of each protein relative to the initial structures.

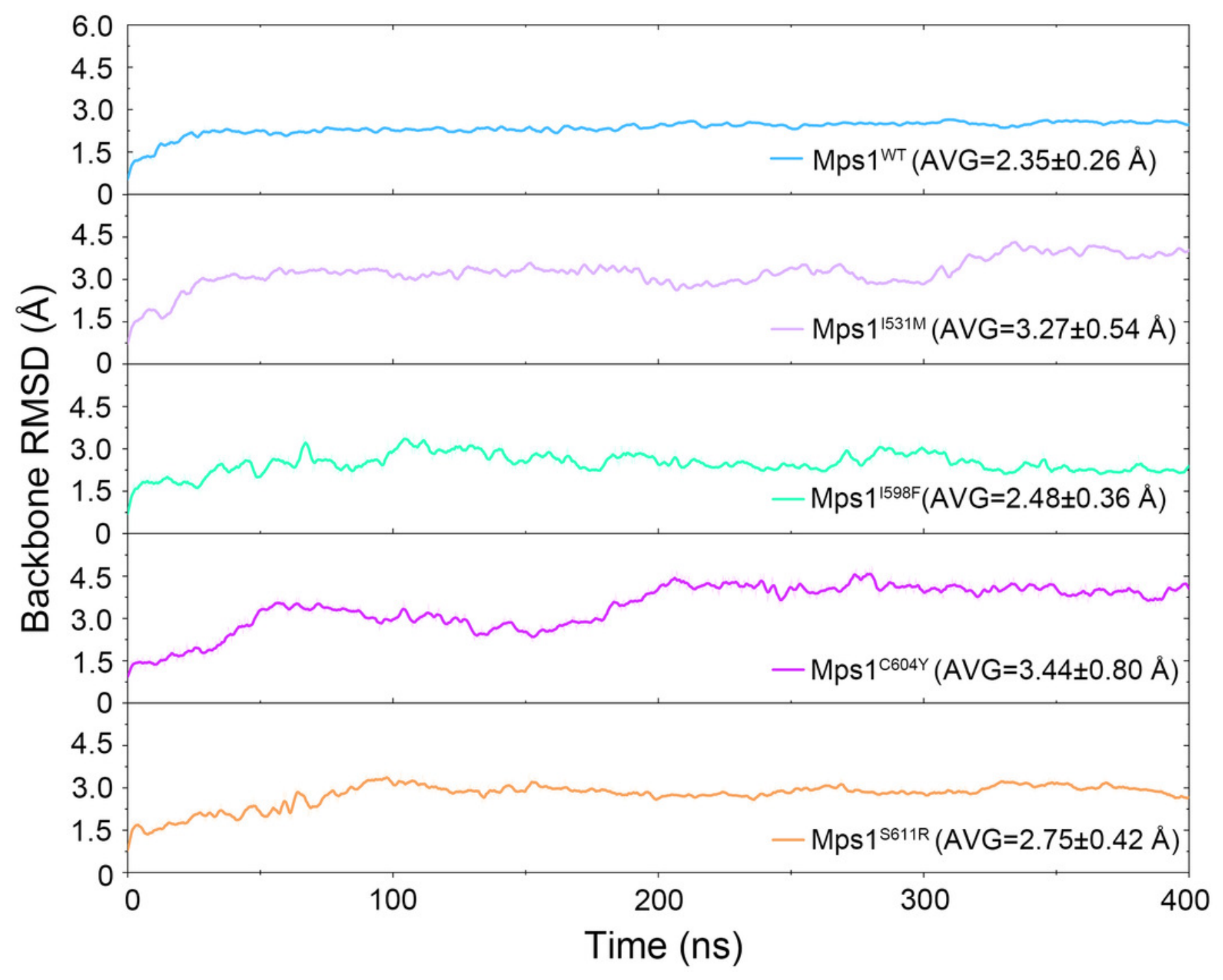


Figure 8

Time evolution of the RMSD values of Cpd-5 in the five studied systems from GaMD simulations. The values reflect the equilibration of $\mathrm{Cpd}-5$ in different protein relative to the initial structures.

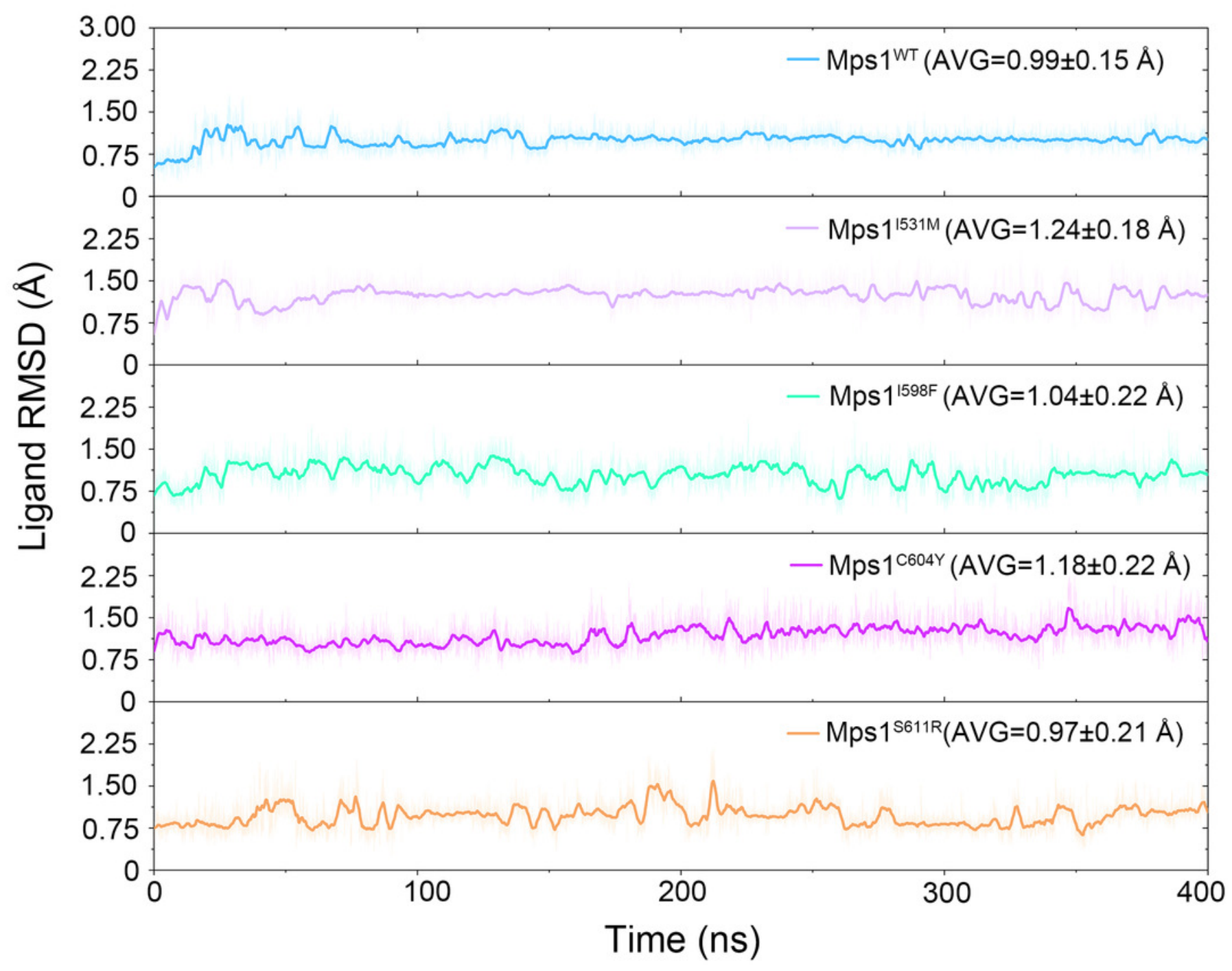


Figure 9

Alignment of the last snapshot between $M p s 1^{\mathrm{WT}} / \mathrm{cpd}-5$ and the mutant systems from GaMD simulations.

(A) Mps1 ${ }^{\mathrm{WT}} / \mathrm{cpd}-5$ (lime) and Mps1 $1^{1531 \mathrm{M}} / \mathrm{Cpd}-5$ (magenta). (B) Mps1 ${ }^{\mathrm{WT}} / \mathrm{cpd}-5$ (lime) and Mps1 $1^{1598 F} / C p d-5$ (magenta). (C) Mps1 $1^{\text {WT}} /$ cpd-5 (lime) and Mps1 $1^{\mathrm{C} 604 \mathrm{Y}} / \mathrm{Cpd}-5$ (magenta). (D) Mps1 ${ }^{\mathrm{WT}} / \mathrm{cpd}-5$ (lime) and Mps1 ${ }^{\mathrm{S} 611 \mathrm{R}} / \mathrm{Cpd}-5$ (magenta).

A

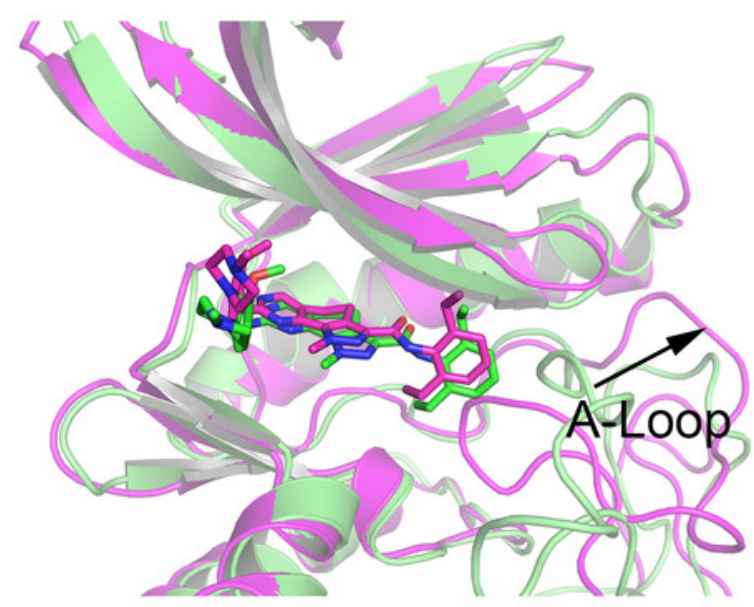

C

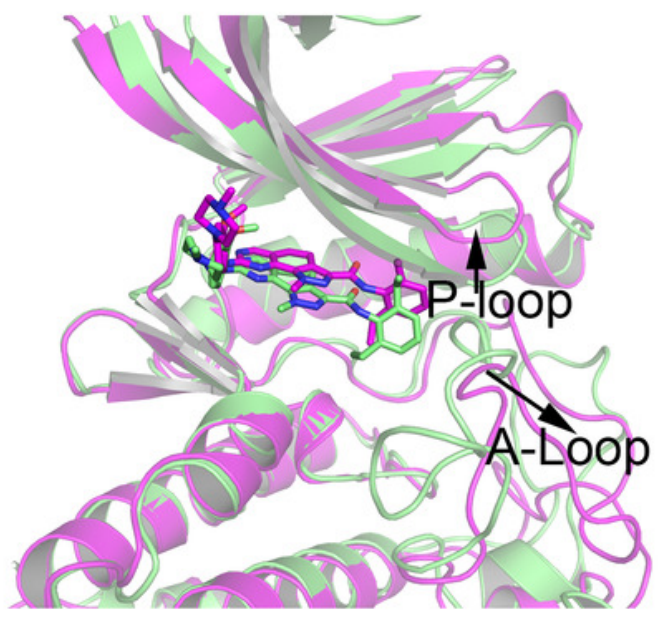

B

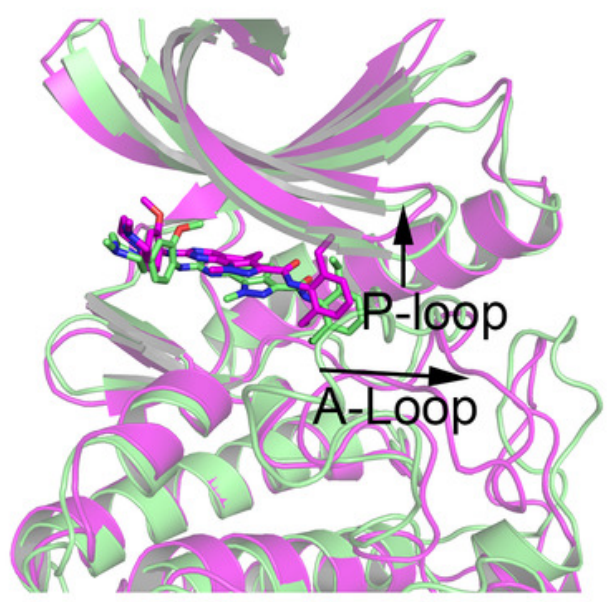

D

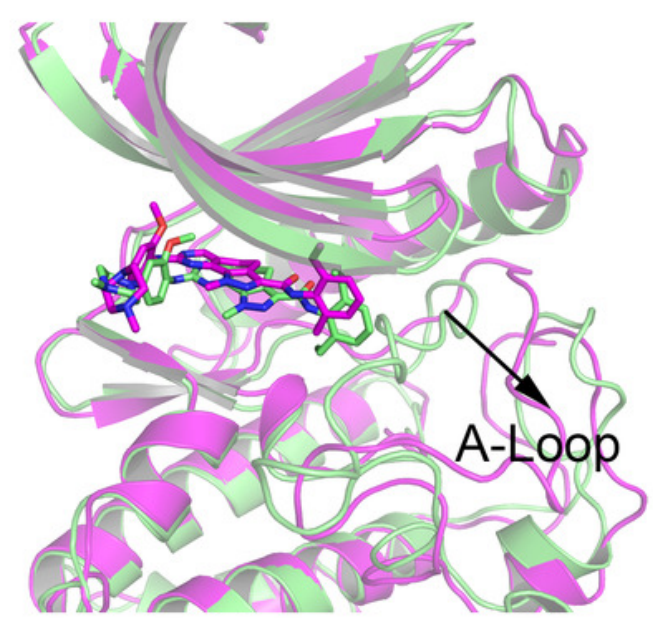


Figure 10

PCA scatter plot of 50,000 snapshots from GaMD simulations along the first two principal components and plotted against time.

(A) Mps1 ${ }^{\mathrm{WT}} / \mathrm{cpd}-5$. (B) Mps1 1531M/Cpd-5 (magenta). (C) Mps1 $1^{1598 \mathrm{~F}} / \mathrm{Cpd}-5$. (D) Mps1 ${ }^{\mathrm{C} 604 \mathrm{r}} / \mathrm{Cpd}-5$; (E) $\mathrm{Mps1}^{\mathrm{S611R}} / \mathrm{Cpd}-5$.

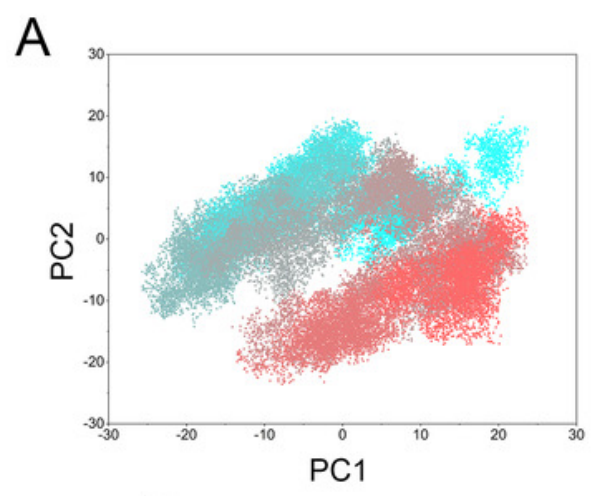

D

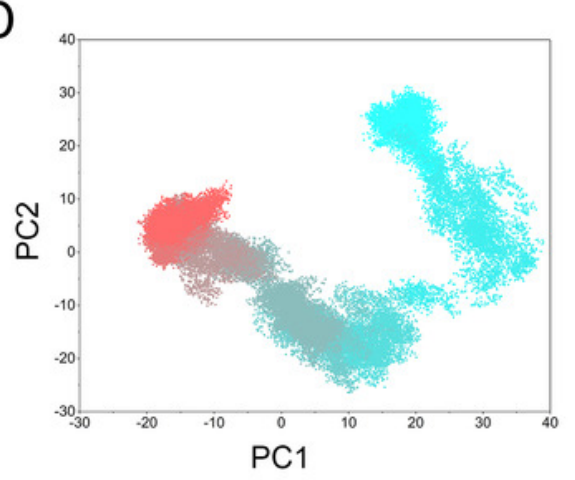

B

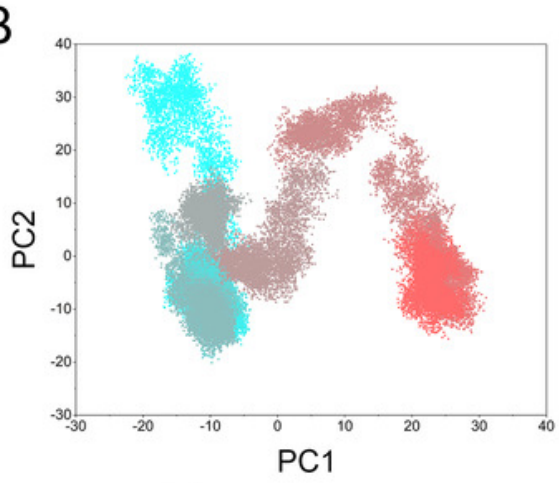

$\mathrm{E}$

E

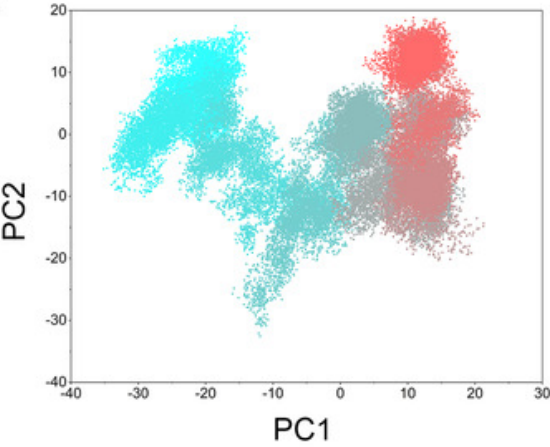

C
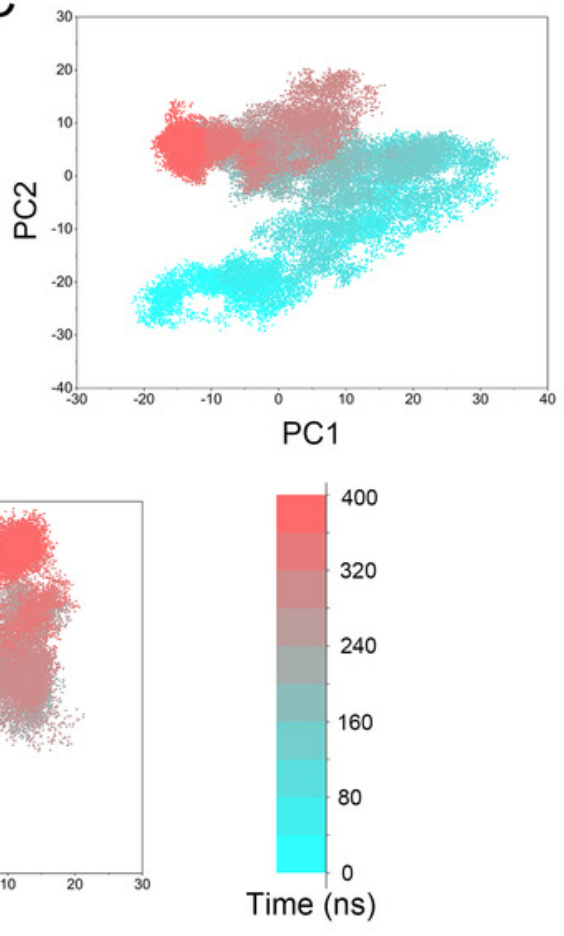
Figure 11

Two-dimensional free energy landscape of the first and second principal components calculated from GaMD simulations.

(A) Mps1 ${ }^{\mathrm{WT}} / \mathrm{cpd}-5$. (B) Mps1 $1531 \mathrm{M} / \mathrm{Cpd}-5$ (magenta). (C) Mps1 $1^{1598 \mathrm{~F}} / \mathrm{Cpd}-5$. (D) Mps1 ${ }^{\mathrm{C} 604 \mathrm{r}} / \mathrm{Cpd}-5$. (E) $\mathrm{Mps1}^{\mathrm{S611R}} / \mathrm{Cpd-5}$.

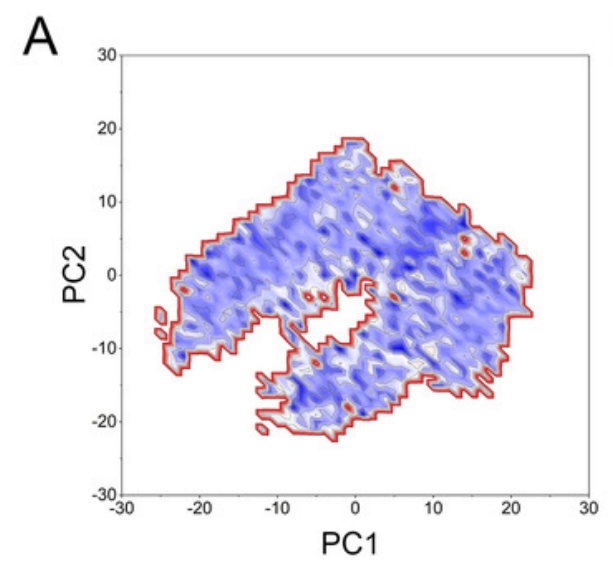

D



B

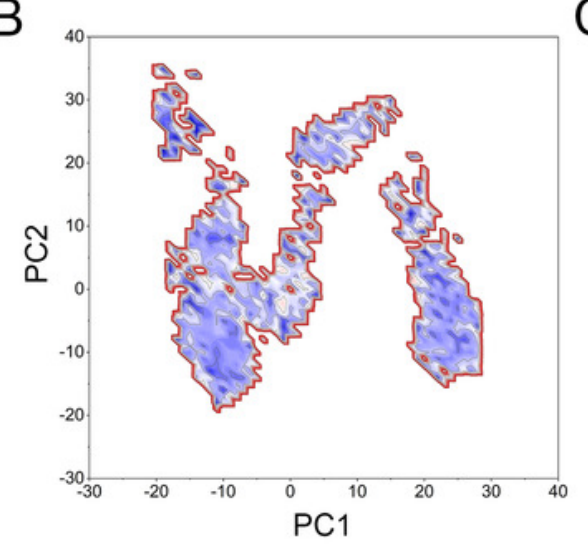

E

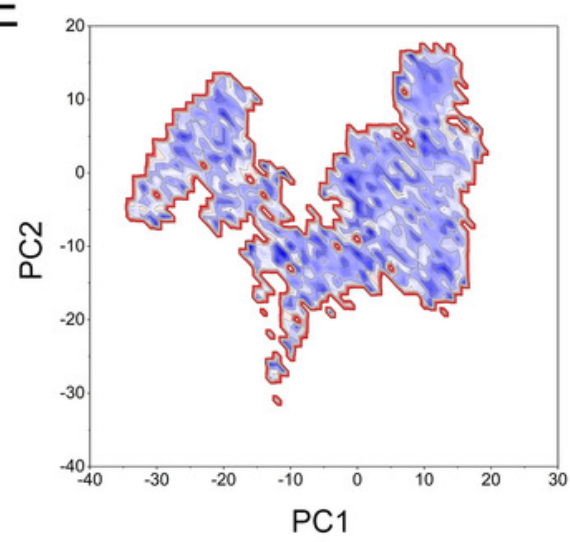

C
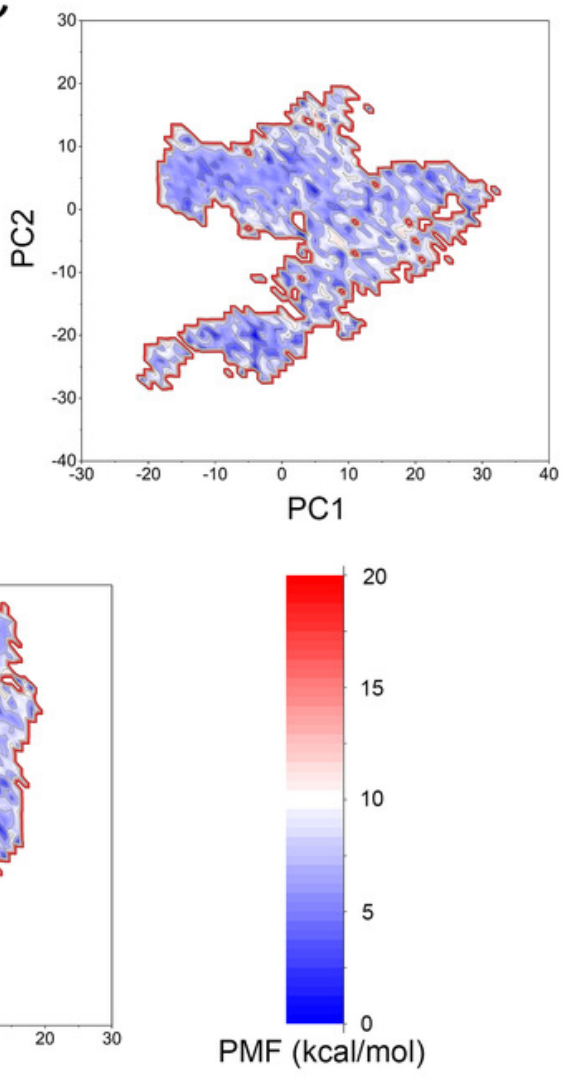


\section{Table $\mathbf{1}$ (on next page)}

Binding free energies of Cpd-5 in WT and mutant systems $(\mathrm{kcal} / \mathrm{mol})$ 


\begin{tabular}{lllllll}
\hline Name & $\Delta E_{\mathrm{vdW}}$ & $\Delta E_{\text {elec }}$ & $\Delta G_{\mathrm{GB}}$ & $\Delta G_{\mathrm{SA}}$ & $\Delta G_{\text {calc }}$ & $\mathrm{IC}_{50}(\mathrm{nM})$ \\
\hline $\mathrm{Mps}^{\mathrm{WT}}$ & $-73.57 \pm 4.62$ & $-26.98 \pm 7.14$ & $49.74 \pm 5.95$ & $-8.92 \pm 0.48$ & $-59.73 \pm 4.72$ & 33.2 \\
$\mathrm{Mps}^{\mathrm{I531M}}$ & $-62.15 \pm 3.78$ & $-27.71 \pm 7.11$ & $47.89 \pm 5.56$ & $-7.91 \pm 0.41$ & $-51.89 \pm 4.14$ & 119.0 \\
$\mathrm{Mps}^{\mathrm{I598F}}$ & $-63.28 \pm 5.09$ & $-28.04 \pm 7.15$ & $47.37 \pm 5.66$ & $-7.72 \pm 0.55$ & $-52.00 \pm 4.61$ & 72 \\
$\mathrm{Mps}^{\mathrm{C} 604 \mathrm{Y}}$ & $-61.16 \pm 3.89$ & $-30.23 \pm 5.44$ & $49.61 \pm 4.63$ & $-7.98 \pm 0.36$ & $-49.77 \pm 3.67$ & 374.8 \\
$\mathrm{Mps} 1^{\mathrm{S} 611 \mathrm{R}}$ & $-66.43 \pm 4.28$ & $-27.79 \pm 6.75$ & $47.75 \pm 4.51$ & $-8.18 \pm 0.42$ & $-54.66 \pm 3.74$ & 129.9 \\
\hline
\end{tabular}

$1 \Delta E_{\mathrm{vdW}}$ : Van der Waals energy; $\Delta E_{\text {ele }}$ : Electrostatic energy; $\Delta G_{\mathrm{GB}}$ : Electrostatic contribution to solvation;

$2 \Delta G_{\mathrm{SA}}$ : Non-polar contribution to solvation; $\Delta G_{\text {calc: }}$ Binding free energy; $\mathrm{IC}_{50}$ : half maximal inhibitory concentration. 